\title{
Performance Evaluation on EZW \& SPIHT Image Compression Technique
}

\author{
jyoti Vitthal Chhatrband, Bhagwat kakade \\ '(Electronics and Telecommunication, RKDF college of Science \& Technology/RGPV, India) \\ ${ }^{2}$ (Electronics and Telecommunication, RKDF college of Science \& Technology/RGPV, India)
}

\begin{abstract}
In image processing algorithms the wavelet transform is widely used in image processing algorithms. In this paper two wavelet compression techniques are applied on the image. The image compression is performed using EZW and SPIHT wavelet based compression techniques. A good flexibility to a wide range of data have presented in wavelet compression, while being of reasonable complexity. These techniques are more efficient and provide a better quality in the image. The different techniques are compared by using the performance parameters (1)Peak Signal to Noise Ratio (PSNR), (2) Mean Square Error (MSE), (3) Compression Ratio (CR) and (4) Bit rate (BR) at different threshold value. These parameters are observed to know which technique is better for image compression in wavelet. It is observed that compression ratio for SPIHT is better as compared to compression ratio of EZW.
\end{abstract}

Keywords: EZW, MSE, PSNR,SPIHT.

\section{Introduction}

Images contain large amount of information that creates problems for storage and network communication. The main objective of Image compression is to reduce this trivial data and store the image in a more efficient manner. It is advantageous to compress the image by storing only the essential information needed to reconstruct the image but it is easy to compress the transformed image rather than compressing the original image. Images can be thought of large array of pixel value, each pixel giving intensity values at that particular point. Therefore images having large and redundancies, and conversely images that have frequent and large changes in colour will be less redundant and harder to compress.

Compression is an asset in communication as it reduces the amount of data that is required to be sent. Since the revolution of media mobile phones sending data to different networks on a limited bandwidth has been a concern. Tremendous improvements in VLSI over the decade have bought smaller Charge Coupled Device (CCD) and hence higher resolution images. This has led the industry to find compression schemes to compress these images and hence we have higher resolution image which does not take much storage. Waveletbased techniques are the latest development in the field of image compression which offers multi resolution capability leading to superior energy compaction with high quality reconstructed images at high compression ratios. This transformed image is stored and we need to recover the image an inverse transform is applied and we get the original image back. Wavelet Transform is used to achieve higher compression ratio while maintaining the image quality.

\section{Identify, Research And Collect Idea}

\section{Wavelet And Image Compression}

Wavelet analysis can be used to divide the information of an image into approximation and detail subsignals. Image compression having advantage of redundancies and reduces the overall size of the image so the image can be stored and transmitted efficiently. Image Compression technique is divided into two techniques lossless image Compression and Lossy Image Compression. The amount of information retained by an image after compression and decompression is known as the "energy retained". If the energy retained is $100 \%$ then the compression is known as "lossless", as the image can be reconstructed exactly. If any values are changed then energy will be lost and known as "lossy" compression. Lossless

In Medical and Technical images compression is used. Whereas, Lossy Compression is used in natural images, images that needs to be sent on a communication network. Wavelet means "Small Wave" Wavelet Transform like Fourier Transform converts the time signal to a frequency signal using a set of basic functions. Wavelet unlike Fourier transform which uses trigonometric polynomials uses wavelets which are form from expansions and dilations of the Mother Wavelet. These wavelets have an added advantage in scalability property both in frequency and time domain. This permits a closer connection between the coefficients generated and the functions. Numerical stability and better manipulation is ensured. It can be shown that every Fast Fourier Transform (FFT) coefficients can be formulated using wavelet analysis providing better spatial and frequency information. Wavelet analysis has overcome lot of difficulties which were faced while using Fourier 
analysis such as how to relate the Fourier coefficients to a local scale. Wavelet is having an average value of zero and it can be defined over a finite interval. The process behind the wavelet transform is any arbitrary function $(t)$ can be defined in the form of a superposition of a set of such wavelets or basis functions. These basis functions are simply called as the baby wavelets. These baby wavelets are obtained from the mother wavelets by scaling (contractions) and shifts (translations). [1] [2] [3].

The typical Wavelet Compression and Decompression system as shown in figure 1 and figure 2.

Wavelet analysis can be used to divide the information of an image into approximation and detail sub-signals. The approximation sub-signal shows the vertical, horizontal and details or changes in the image. If these details are very small then they can be set to zero without significantly changing the image.

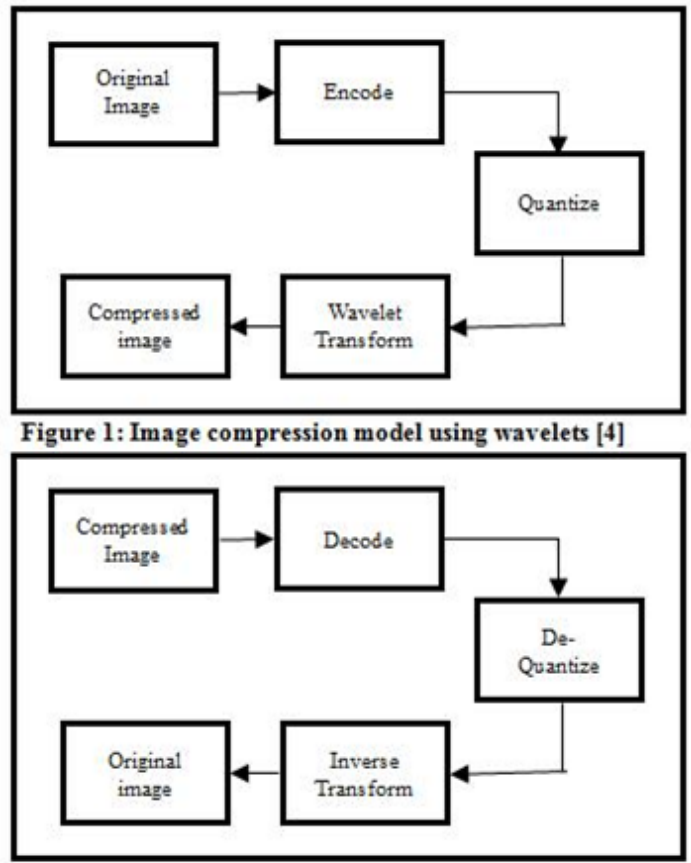

Figure2: Image decompression model using wavelets [4]

Wavelet Compression techniques takes advantage of this zeros as they can be eliminated without much loss to the quality of the image

\subsection{EZW (Embedded Zero- trees of Wavelet Transform)}

EZW proposed by J.M. Shapiro is a first lossy image compression algorithm which shows full power of wavelet based compression. It is simple, effective algorithm, having the property that the bits in the bit stream are generated in order of importance, yielding a fully embedded code [2]. The EZW algorithm is based on four principal concepts that is discrete wavelet transform or hierarchical sub-band de-composition, prediction of the absence of significant information across scales, entropy coded successive-approximation quantization, data compression using Arithmetic or Huffman coding.

\subsubsection{Terms in EZW:}

Embedded -An embedded coding is a process of encoding the transform magnitudes that allows for progressive transmission of the compressed image.

Zero-tree -Zero tree are a concept through which compression is achieved by allowing for a concise encoding of the positions of significant values that result during the embedded coding process.

A wavelet transform transforms a signal from the time domain to the joint time-scale domain. This means that the wavelet coefficients are two-dimensional. If we want to compress the transformed signal we have to code not only the coefficient values, but also their position in time. When the signal is an image then the position in time is better expressed as the position in space. After wavelet transforming an image we can represent it using trees because of the sub sampling that is performed in the transform. A coefficient in a low sub band can be thought of as having four descendants in the next higher sub-band (see figure 1). The four descendants each also have four descendants in the next higher sub-band and we see a quad-tree emerge: every root has four leafs. The EZW encoder is based on two important observations [5]: 1. Progressive encoding is used for compressive wavelet transformed images, since the higher sub-band only add detail. 2. Large wavelet coefficients are more important than small wavelet coefficients. 
2.1.2. EZW Algorithm

The EZW Algorithm have the following steps.

Step 1 (Initialization)

$T_{0}=2^{\left|\log _{2} C_{\max }\right|}$

Threshold is initialized by where $\mathrm{C}_{\max }$ is the maximum of the absolute value of the DWT coefficients. In EZW algorithm entire wavelet coefficient matrix is scanned in order to find out the maximum value of the coefficient [6]. We can infer from the computed coefficients for any image that maximum value of coefficient occurs at the lowest frequency sub band (LL band). We see that two passes are used to code the image. In the first pass, the dominant pass, the image is scanned and a symbol is outputted for every coefficient [7].

\section{Step 2 (Dominant pass)}

Coefficients on Dominant List are compared to $\mathrm{T} 0$ and their significance map is made.

The significance map can be efficiently represented as a string of symbols from a 4 symbols are used:

1. Zerotree root $(\mathbf{t})$ : If its absolute value is lower than threshold but one of its children has an absolute value higher than the threshold.

If $\left(\left|\mathrm{x}_{\mathrm{WT}}\right|<\mathrm{T}_{\mathrm{i}}\right) \& \&$ (all descendants of $\left.\mathrm{x}_{\mathrm{WT}}<\mathrm{T}_{\mathrm{i}}\right)$

2. Isolated zero $(\mathbf{z})$ : if its absolute value is lower than the threshold and all of its children have absolute values lower than the thresholld.

If $\left(\left|\mathrm{x}_{\mathrm{WT}}\right|<\mathrm{T}_{\mathrm{i}}\right) \&\left(\left(\right.\right.$ some descendants of $\left.\mathrm{x}_{\mathrm{WT}}>\mathrm{T}_{\mathrm{i}}\right) \|\left(\mathrm{x}_{\mathrm{WT}}\right.$ is the last item)).

3. Significant positive (p): if it is positive and has an absolute value higher than the threshold. If $\left(\left|x_{W T}\right|>=T_{i}\right) \& \&\left(x_{W T}>0\right)$

4. Significant negative $(\mathbf{n})$ : if it is negative and has an absolute value lower than the threshold. If $\left(\left|x_{W T}\right|>=T_{i}\right) \& \&\left(x_{W T}<0\right)$

\section{Step 3 (Subordinate pass)}

All the values in the subordinate list are refined. This gives rise to some juggling with uncertainty intervals and it outputs next most significant bit of all the coefficients in the subordinate list. If the value is greater than the current threshold then a 1 is sent, if less than the threshold a zero is sent. The current threshold is then divided by two and the process is repeated till the threshold doesn't reach the minimum bit value or zero. The decoding used is Huffman decoding scheme. The code also involves the decoding of the coded EZW image to prove the validity of the encoding.

\section{Step 4 (New threshold)}

We calculate the new threshold if the minimum threshold or the desired compression ratio is attained, we stop; if not we repeat stages 2,3 and 4 .

\section{Write down Your Studies and Findings}

\subsubsection{Implementation}

The EZW Algorithm was implemented using MATLAB.

\subsubsection{Initialization}

The image is first read in MATLAB and its pixel values are stored. Than a Discrete Wavelet Transform is done on the image of level 4 . The following diagram gives an example. Now we have $\mathrm{C}_{\max }=1255$.

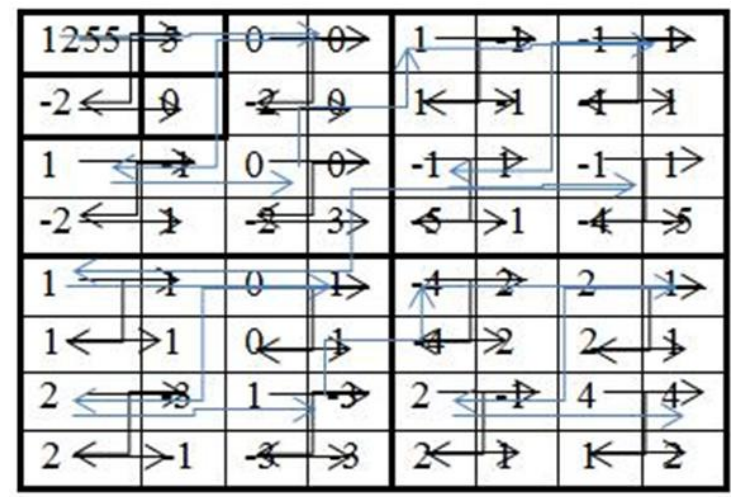

\subsubsection{Dominant Pass:}

The image is then compared with the threshold. The coefficients are obtained using Morton scanning The encoded data of the image is shown below. ppppptppppztztz..... 


\subsubsection{Masking.}

For obtaining Zero tree coefficients we have used an algorithm for masking the coefficients to check whether it is a zero tree or an isolated zero. If during the Morton scan if a value less than threshold is encountered then we place a mask on the daughter trees. The values of the mask are compared with the threshold and if they're less than the threshold then the pass is a zero tree pass if not then it is an isolated zero.

\subsubsection{Subordinate pass:}

The Dominant pass is passed through a refinement pass. This converts the dominant pass values into bit format. The previous values have to be stored to be read again and added to the new values. An example is shown below.

$11100011100101011 \ldots \ldots$

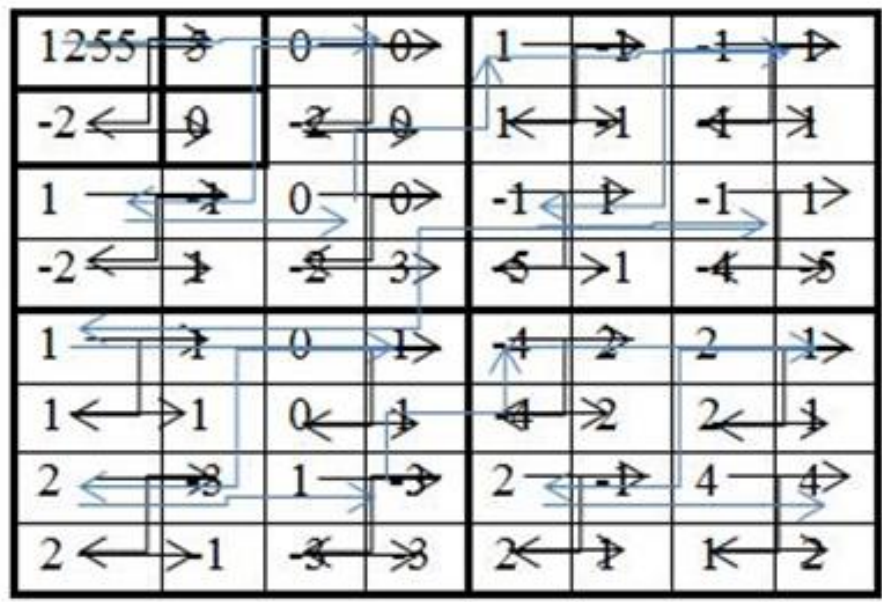

\begin{tabular}{|l|l|l|l|l|l|l|l|}
\hline 1255 & 5 & 0 & 0 & 1 & -1 & -1 & 1 \\
\hline-2 & 0 & -2 & 0 & 1 & -1 & -1 & 1 \\
\hline 1 & -1 & & 0 & -1 & 1 & -1 & 1 \\
\hline-2 & 1 & -8 & 3 & -5 & -1 & -4 & -5 \\
\hline 1 & 1 & 0 & 1 & -4 & 2 & 2 & \\
\hline 1 & 1 & 0 & 1 & - & 2 & 2 & 1 \\
\hline 2 & -3 & 1 & -3 & 2 & -1 & 4 & 4 \\
\hline 2 & -1 & -3 & -3 & 2 & 1 & 1 & 2 \\
\hline
\end{tabular}

\subsection{PIHT (Set partitioning in Hierarchical tress)}

SPIHT algorithm is refined version of EZW algorithm proposed by A. Said, Pearlman [8]. SPIHT stands for set partitioning in Hierarchical tress. Hierarchical tress refers to the quad-tress as defined in in EZW. The SPIHT coding is an improved version of the EZW algorithm that achieves higher Compression and better performance than EZW [9]. Initially we apply wavelet transform on the image and obtain the wavelet coefficient .Then coding is applied on the obtained coefficient. Set partitioning refer to the way these quad-trees divide up, partition and these coefficient at a given threshold. Thus SPIHT provides the best results of PSNR at higher ratios than EZW due to its partitioning property which increases its compressive power. Here the coding algorithm uses three lists called List of Significant Pixels (LSP), List of Insignificant Pixels (LIP) and List of Insignificant Sets (LIS).

LIS (list of insignificant sets): LIS contains set of wavelet coefficient which is defined by tree structures, and which had been found to have magnitude smaller than a threshold (are insignificant). The sets exclude the coefficient to the tree or all sub-tree roots and have at least four elements.

LIP (list of insignificant pixel): LIP contains individual coefficient and have magnitude smaller than the 
threshold.

LSP (list of significance pixel): pixel found to have magnitude larger than the threshold.

As an initialization step, the number (n) of magnitude refinement passes that will be necessary is determined from the maximum magnitude of the coefficient. Initially all pixels are treated as insignificant. The initialization is followed by three major passes-the sorting pass, the magnitude refinement pass and the quantization step update pass which are iteratively bits are transmitted.

During the sorting pass, the pixels in the LIP, which are insignificant till the previous pass, are tested, and those that become significant are moved to the LSP. Similarly the sets in LIS are examined in order for significance and those which are found to be significant are moved from the list and partitioned. The new subsets with more than one element are added to the LIS and the single pixels are added to LIP or the LSP, depending upon their significance. During the magnitude refinement pass, the pixels in the LSP are encoded for nth MSB.

\subsubsection{Spiht Algorithm}

Notations used in the algorithm are defined as follows:

$\mathrm{O}(\mathrm{i}, \mathrm{j})$ : set of coordinates of all offspring of node $(\mathrm{i}, \mathrm{j})$; children only

$\mathrm{D}(\mathrm{i}, \mathrm{j})$ : set of coordinates of all descendants of node $(\mathrm{i}, \mathrm{j})$; children, grandchildren, great-grand, etc.

$\mathrm{H}(\mathrm{i}, \mathrm{j})$ : set of all tree roots (nodes in the highest pyramid level); parents

$\mathrm{L}(\mathrm{i}, \mathrm{j}): \mathrm{D}(\mathrm{i}, \mathrm{j})-\mathrm{O}(\mathrm{i}, \mathrm{j})$ (all descendants except the offspring); grandchildren, great-grand, etc.

Step 1 (Initialization)

$\mathrm{n}=\log 2(\max \mid$ coeff $\mid)$ LIP $=$ All elements in H LSP = Empty

LIS $=$ D's of Roots

Step 2 (Sorting Pass)

Step-2.1: For each entry in LIP, output the significance ("1" if significant " 0 " if not significant).If found significant remove it from the LIP and add to the LSP.

Step-2.2: For each entry in LIS, output the significance. If found significant output its sign.

Depending upon whether it is the $\mathrm{D}(\mathrm{n} 1, \mathrm{n} 2)$ set or the $\mathrm{L}(\mathrm{n} 1, \mathrm{n} 2)$ set perform the partitioning as:

If $D(i, j)$ is found significant, partition it into $L(i, j)$ plus four single element sets wih

$(i, j) \in O(i, j)$.

If $L(i, j)$ is found significant, partition it into four set of $D(i$,

j) $\in \mathrm{O}(\mathrm{i}, \mathrm{j})$.

According to the significance, update the LIS, LIP and LSP.

Step 3 (Refinement pass)

For each element in LSP-except those just added above output the nth most significant bit of coefficient.

End loop over LSP

\section{Fig SPIHT Sorting Pass}

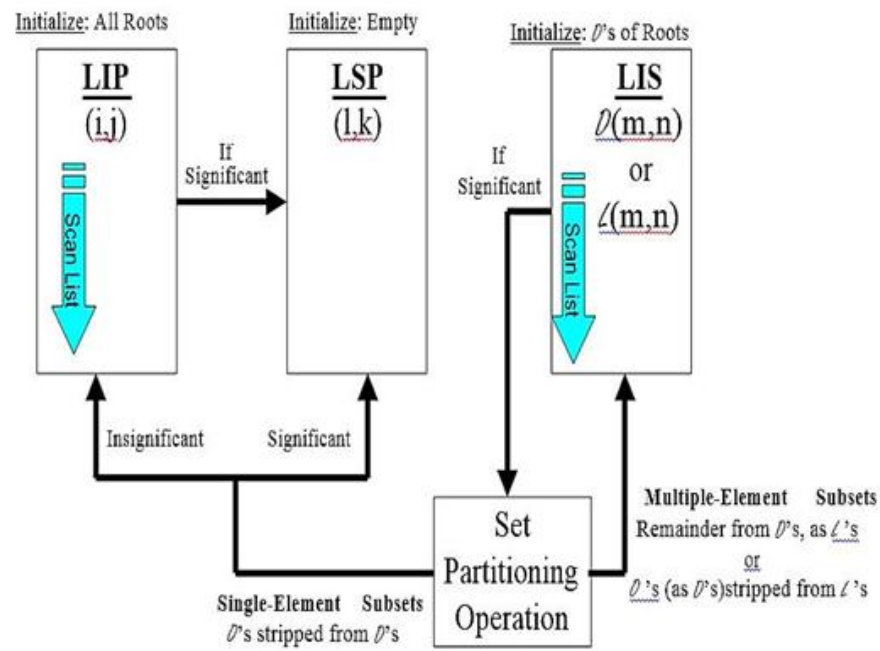

Step 4 (Quantization state update) Decremented by 1

Steps 2, 3 and 4 are repeated until $n=0$

It is the characteristic of SPIHT algorithm that it generally operates on an entire image at once.

Due to this the size of the three lists is often quite big and takes a lot of memory. The whole image is loaded and 
transformed, and then the algorithm requires repeated access to all lists [10].

2.2.2 Implementation (SPIHT)

Step 1 (Initialization)

The SPIHT parameters are initialized. $\operatorname{LIP}=[(2,3)(4,1)(2,3) \ldots]$

$\mathrm{LIS}=[(0,0)(0,0)(0,0) \ldots] \mathrm{LSP}=[]$

Step 2 (Sorting Pass)

In the sorting pass each bit is compared with the threshold and stored in the respective list. A bit counter is assigned to stop the process once the limit has reached. The specific bit is removed from LIP and added to LSP. $\mathrm{LIP}=[(2,4)(3,2)(4,2)(5,1)(5,3)(5,4) .$.

$\mathrm{LIS}=[(8,1)(1,5)(1,11)(1,12)(2,11)(2,12) . ..] \mathrm{LSP}=[(1,2)(1,1)(2,1)(2,2)(1,3)(3,1) .$.

Step 3 (Refinement Pass)

The LSP list is used and for each value is refined. The value of 256, 14 , and 7 are headers for the bit stream.

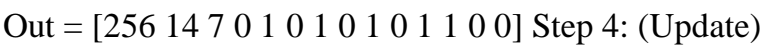

Updating the threshold value $B$. Results

The results are found out by performing the compression and decompression on the image using MATLAB.

\section{$2.3 \mathrm{EZW}$}

At different threshold the original Image and its wavelet transform along with the compressed image are given below. As the results shows, the PSNR increase as the threshold keeps

decreasing and the MSE keeps reducing as the threshold values is reduced.

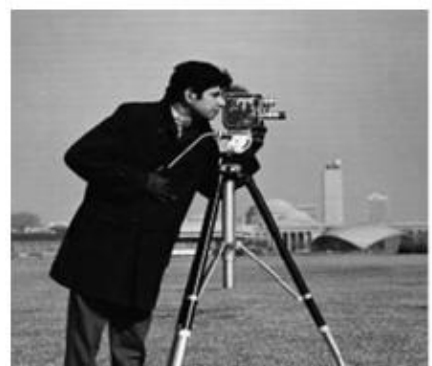

Original Image

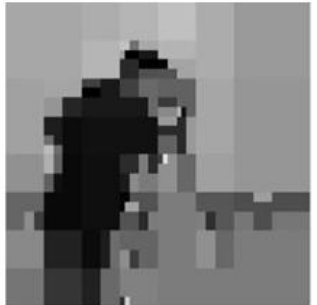

Threshold: $\mathbf{5 1 2}$

PSNR: 18.803

MSE: 961.0625

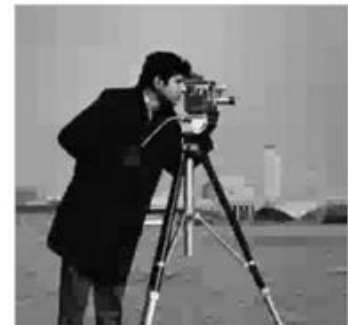

Threshold: 64

PSNR: 26.7446

MSE: 137.6015

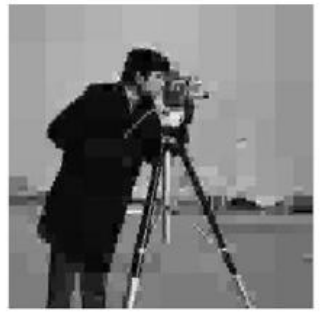

Threshold: 128

PSNR: 23.1695

MSE: 313.4029

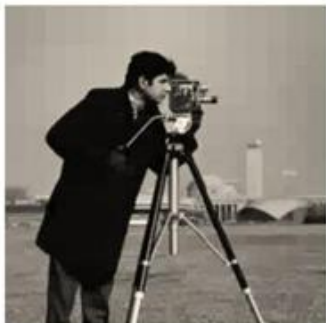

Threshold: 32

PSNR: 30.7411

MSE: $\mathbf{5 4 . 8 2 3 5}$

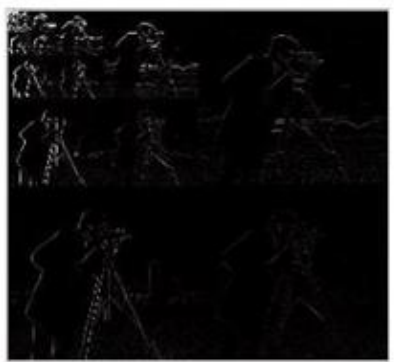

Wavelet Transform

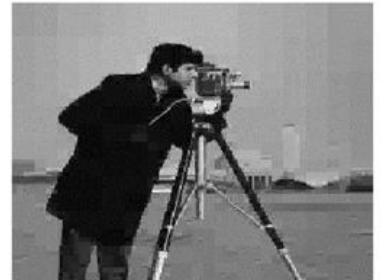

Threshold : 64

PSNR : 25.849

MSE: 169.10

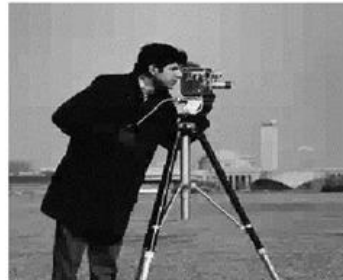

Threshold : 32

PSNR : 29.95

MSE: 65.75

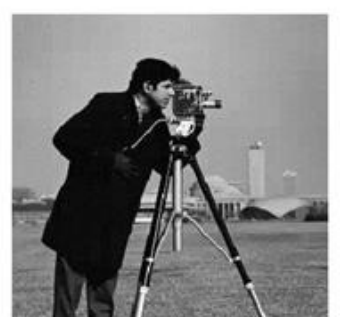

Threshold: 16

PSNR : 34.977

MSE: 20.67

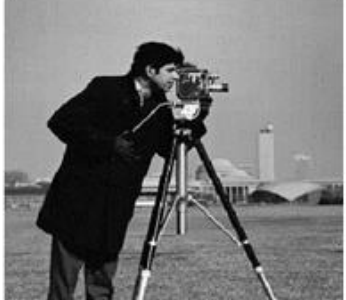

Threshold : 8

PSNR : 39.88

MSE: 6.68 


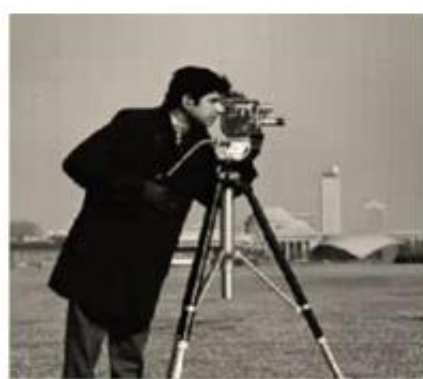

Threshold: 16

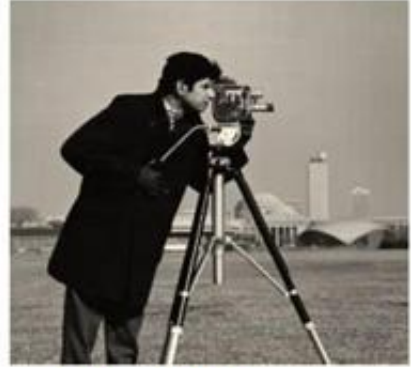

Threshold: 8

Table no. 1: Comparison of various values for EZW and SPIHT

\begin{tabular}{|l|l|l|l|l|l|l|l|l|}
\hline \multirow{2}{*}{ Threshold } & \multicolumn{4}{|c|}{ EZW } & \multicolumn{4}{c|}{ SPIHT } \\
\cline { 2 - 10 } & PSNR & MSE & BR & CR & PSNR & MSE & BR & CR \\
\hline 8 & 40.56 & 5.718 & 0.026 & 3.46 & 39.9 & 6.68 & 0.089 & 5.16 \\
\hline 16 & 35.52 & 18.24 & 0.016 & 5.61 & 35 & 20.67 & 0.048 & 8.089 \\
\hline 32 & 30.74 & 54.82 & 0.009 & 10.5 & 30 & 65.75 & 0.023 & 14.85 \\
\hline 64 & 26.74 & 137.6 & 0.004 & 22 & 25.8 & 169.1 & 0.009 & 31.87 \\
\hline 128 & 23.17 & 313.4 & 0.002 & 52.6 & 22.6 & 359.5 & 0.003 & 78.1 \\
\hline 512 & 18.8 & 961.1 & 0.0002 & 435 & 18 & 1040 & 0.001 & 675.6 \\
\hline
\end{tabular}

\section{Compression Ratio for EZW and SPIHT}

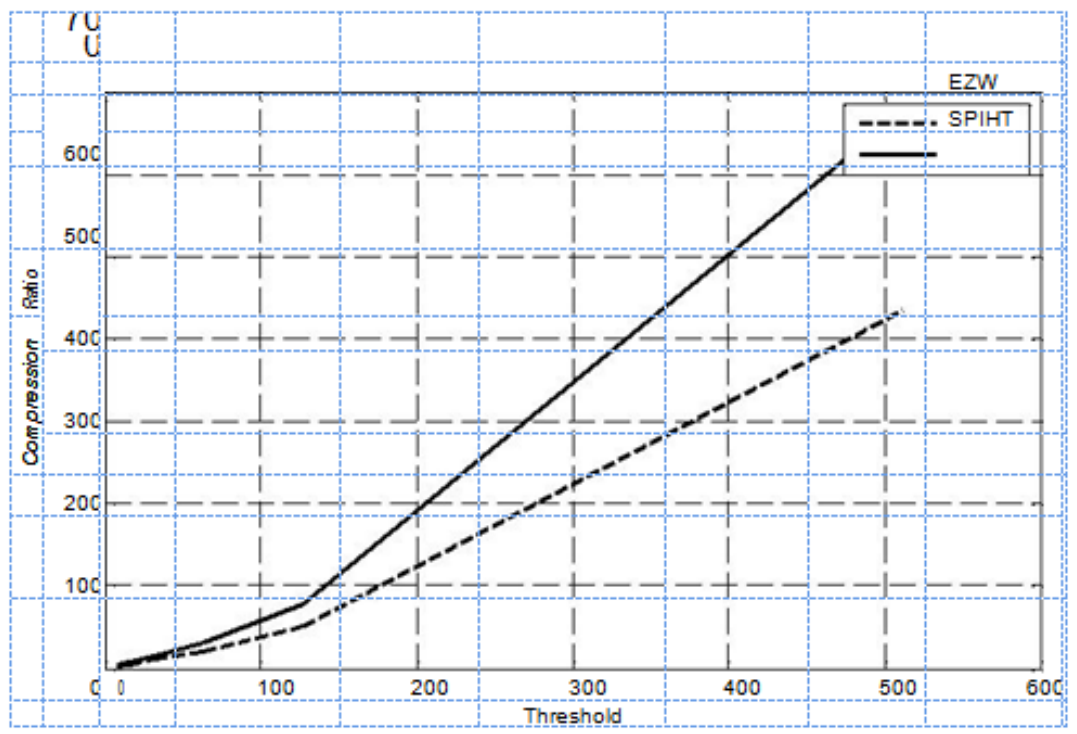

Fig 5.4 Compression ratio for EZW and SPIHT

\section{Conclusion}

In this paper we have implemented two efficient algorithms EZW and SPIHT which have some common strength such as, depending on the requirement of the application, the encoding and decoding can be stopped and the desired output quality of image can be retrieved. Also each algorithm gives a significant data compression. After implementing EZW \& SPIHT algorithms we found following results:

\subsection{EZW:}

For threshold $8,16,32,64,128,512$ the respective bit rates are $0.0257,0.0160,0.0086,0.0041$, $0.0017,0.0028$ and the PSNR are 40.5582, 35.5198, 30.7411, 26.74, 23.16 and 18.80. We can see as the threshold increases the bit-rate goes on decreasing and the decreases in bit-rate is very significant at the same time the PSNR values also decreases and with Compression Ratio (CR). From the graph plotted in figure no. it can be seen that if the compression ratio increases then PSNR increases and MSE decreases.

\subsection{SPIHT:}

For threshold 8, 16, 32, 64, 128, 512 the respective bit rates are $0.0887,0.0483,0.0225,0.0092,0.0031,0.0010$ and the PSNR are 39.88, 34.97, 29.95, 25.84, 22.57 and 17.96. At lower threshold the change in PSNR and bit- 
rate is not much distinguishable as threshold changes from 8 to 16 PSNR changes 0.00887 to 0.0483 .But at higher threshold bit- rates decreases to greater extent and the subjective quality of the image is also degraded.

\section{References}

[1]. Ahmed N. T., Natarajan and K. R. Rao, "On image processing and discrete cosine transform", IEEE Trans. Medical Imaging, vol C-23, pp. 90-93, 1974.

[2]. KP. Soman \& K.I .Ramachandran, "Insight into Wavelets from theory to practice", Prentice Hall India, New Delhi 2002.

[3]. Charilaos Christopoulos, "The JPEG 2000 still image coding system: an overview", IEEE trans. On consumer electronics, vol.46, No.4, PP.1103- 1127, Nov.2000.

[4]. A.R. Calder bank, I. Daubechies, W. Seldens, B.L. YEo, "wavelet transform that map integers to integer", Applied and computational harmonic analysis, vol.5, No.3, July 1998, pp.332-369. (Pubitemid128424411) Said, W.A. Pearlman, "A new fast and efficent image codec based on set partitioning in Hierarchical trees", IEEE Trans. On circuits and systems for video technology, vol.6, No.3, PP.243-250, June.1996

[5]. W.Sweldens., "The lifting scheme: A custom design construction of Bi orthogonal wavelets", Applied and computational harmonic analysis,Vol.3, April 1996, pp.186-200. (Pubitemid 126375198)

[6]. M.Antonini, M.Barlaud, P.Mathieu, I.Daubechies, "Image coding using wavelet transform", IEEE Trans. on image processing, Vol.1, No.2, pp. 205-220, April1992.

[7]. Marta Mrak, Sonja Grgic and Mislav Grgic, "Picture quality measures in image compression systems", IEEE trans. in Electrical and computing. PP. 233-237, 2003.

[8]. Abstract | Full Text: PDF (431KB)|Full Text: HTML

[9]. Mohammed Beladgham, A.Bessaid, A.M.Lakhdar, A.T.Ahmed, "Improving quality of medical image compression using Bi orthonal CDF wavelet based on lifting scheme and SPIHT coding" Serbian Journal of electrical engineering rative analysis", Vol.8, No.2, May-2011, pp.163-179 [CrossRef]

[10]. Khalid Sayood, "Introduction to Data Compression", Third Edition, PP. 423-513. 2011.

[11]. R.C.Gonzalez and Wood, "Digital image processing using MATLAB" Reading, Second addition, McGraw Hill Companies, PP. 331-439, 2011.

[12]. David salomon, "Data compression- the complete reference", 4th Edition, Springer international edition, PP.532-639, 2011.

[13]. A.K.Jain, "Fundamentals of Digital Image Processing", Reading, New Jersey: Prentice Hall Inc., PP. 479 - 533, 1989.

[14]. Ritu Chourasiya and Prof. Ajit Shrivastava, "study of image compression based Transmission algorithm using Spiht for Low bit rate application", An International Journal Advanced (ACIJ), Vol.3, No.6, November 2012. 